\title{
Phase drift compensation between injector linac master oscillator and ring master oscillator for stable beam injection at SuperKEKB
}

\author{
Na Liu, ${ }^{1, *}$ Takako Miura, ${ }^{1,2}$ Toshihiro Matsumoto, ${ }^{1,2}$ Tetsuya Kobayashi, ${ }^{1,2}$ Naoko Iida, ${ }^{1,2}$ \\ Feng Qiu, ${ }^{1,2}$ Shinichiro Michizono, ${ }^{1,2}$ Dai Arakawa, ${ }^{2}$ Hiroaki Katagiri, ${ }^{2}$ \\ Yoshiharu Yano, ${ }^{2}$ and Baiting $\mathrm{Du}^{1}$ \\ ${ }^{1}$ SOKENDAI (The Graduate University for Advanced Studies), \\ Shonan Village, Hayama, Kanagawa 240-0193, Japan \\ ${ }^{2}$ High Energy Accelerator Research Organization, 1-1 Oho, Tsukuba, Ibaraki 305-0801, Japan
}

(Received 8 April 2019; published 26 July 2019)

\begin{abstract}
The SuperKEKB injector linac that includes the positron damping ring (DR) delivers low emittance electron and positron beams to the main rings. The beam injection phase from linac to the rings should be stabilized for long-term stable beam injection. The frequencies of the linac master oscillator (MO) and ring $\mathrm{MO}$ are 571.2 and $508.9 \mathrm{MHz}$, respectively. These two different frequency signals are monitored using the direct sampling technique with a common sampling rate. A phase drift of several degrees is observed between the linac and ring MOs. For stable beam injection, a phase drift compensation system is implemented at linac. The system performance was evaluated by the beam energy stability after the bunch compression system from DR to linac.
\end{abstract}

DOI: 10.1103/PhysRevAccelBeams.22.072002

\section{INTRODUCTION}

The SuperKEKB [1] is a double-ring collider that consists of a high energy ring (HER) of $7 \mathrm{GeV}$ electron, and a low energy ring (LER) of $4 \mathrm{GeV}$ positron. SuperKEKB is upgraded from KEKB aiming at 40 times higher luminosity for seeking new physics beyond the Standard Model. Low emittance beams are produced and delivered by an electron/positron injector linac [2]. As shown in Fig. 1, the injector linac has two electron guns: a photo-cathode radio-frequency (rf) gun for low emittance electron beams and a thermionic gun for positron production. The beams are accelerated by $2856 \mathrm{MHz}$ rf accelerating structures to 7 and $4 \mathrm{GeV}$ for electrons and positrons, respectively. The $1.1 \mathrm{GeV}$ positron damping ring (DR) was constructed in the middle of linac for low emittance beams. The positron beam extracted from the DR is reinjected into the $2856 \mathrm{MHz}$ rf accelerating structures. To shorten the bunch length and precisely put the beam on the phase at $2856 \mathrm{MHz}$, the bunch compression system (BCS) comprising a $2856 \mathrm{MHz}$ accelerating structure and an arc section is placed on the beam transport line from DR to linac (RTL).

\footnotetext{
*liuna@ post.kek.jp

Published by the American Physical Society under the terms of the Creative Commons Attribution 4.0 International license. Further distribution of this work must maintain attribution to the author(s) and the published article's title, journal citation, and DOI.
}

Figure 1 describes the synchronization system for the injector linac, DR, and main rings (MR). The injector linac, $\mathrm{DR}$, and MR have their own independent master oscillators (MO) that belong to the commercial Agilent E8663 series [3]. They are synchronized with a $10 \mathrm{MHz}$ trigger generated by the main MO (MMO) signal of $510 \mathrm{MHz}$ by dividing with 51. The MMO, DR MO, and MR MO are located at the SuperKEKB center control room. The DR $\mathrm{MO}$ and MR MO are operated at $508.9 \mathrm{MHz}$ and the phase drift between them is controlled within $\pm 0.1^{\circ}$ (peak to peak) by a phase-locked loop (PLL) [4]. DR MO and MR $\mathrm{MO}$ are collectively called ring MO (RMO) in this paper. The rf reference signals are distributed to the DR and the MR through optical fibers.

The linac MO (LMO) is operated at $571.2 \mathrm{MHz}$ and located in the linac gallery. The $2856 \mathrm{MHz}$ rf reference signal is generated by the LMO [5]. A $510 \mathrm{MHz}$ signal from MMO is transmitted to linac with the coaxial cable for synchronization. The phase drift of the long coaxial cable is compensated by PLL [6]. However, LMO and RMO are only synchronized with the $10 \mathrm{MHz}$ trigger derived from the MMO but without phase regulation. A phase drift of several degrees is observed between the two MOs by the phase monitor [3]. This MO phase drift significantly affected the beam energy stability after the BCS cavity in RTL because of a five times larger phase for $2856 \mathrm{MHz}$. Furthermore, the beam injection phase tuning from linac to the MR had to be conducted frequently, since beam injection background has been a critical problem for the SuperKEKB Belle II detector [7]. To maintain the stable 


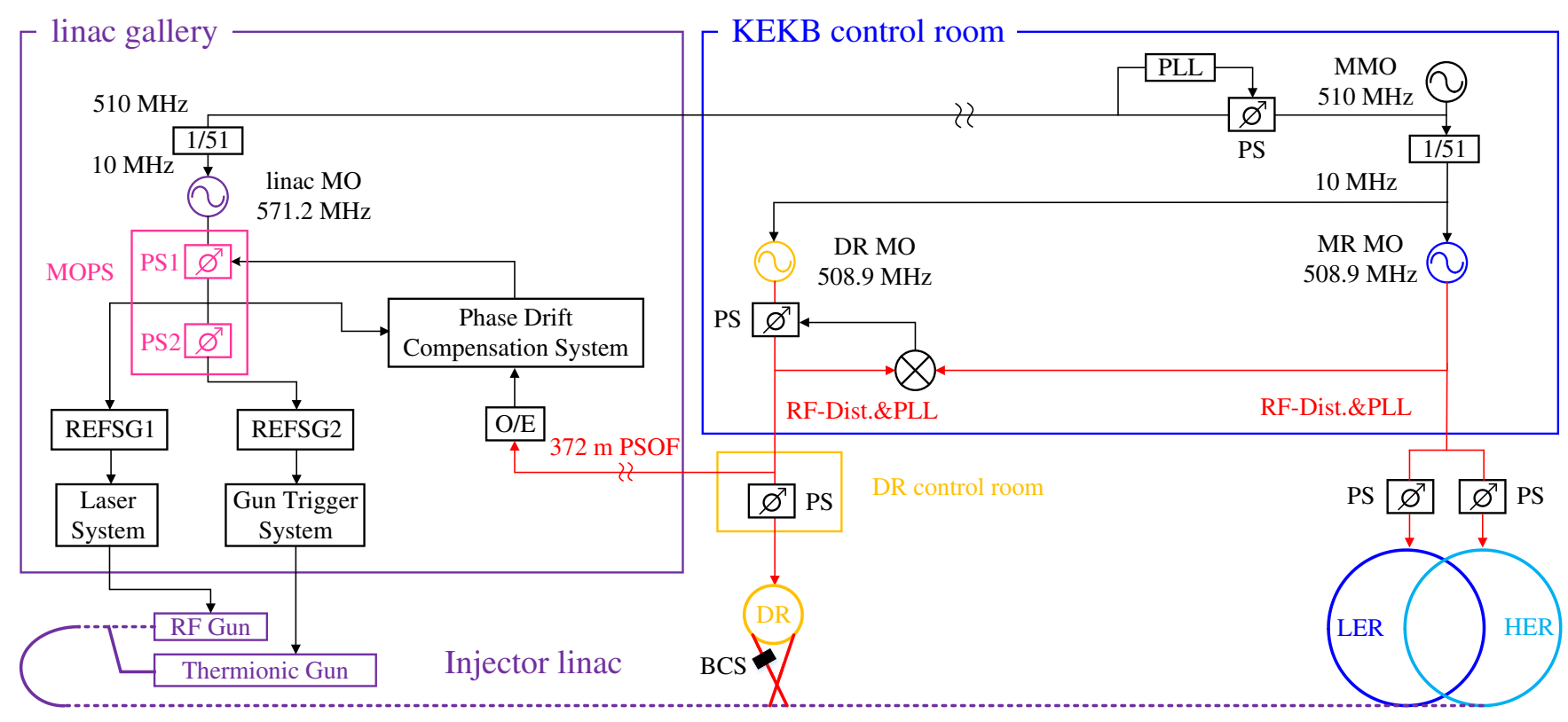

FIG. 1. Block diagram of synchronization system for linac, MR, and DR. PS designates phase shifter; MOPS designates master oscillator phase shifter; REFSG designates reference signal generator; RF-Dist. designates rf reference distribution system; PSOF designates phase stabilized optical fiber; and O/E designates optical/electrical converter.

beam injection, the MO phase drift compensation system is developed. The phase stability between LMO and RMO is required less than $\pm 0.1^{\circ}$ (peak to peak) at $571.2 \mathrm{MHz}$.

\section{DIRECT SAMPLING FOR TWO DIFFERENT FREQUENCY SIGNALS}

To remove the effects of temperature and humidity drift of the measurement system, it is important to make the measurement system common for signals with different frequencies. The direct sampling technique, therefore, is adopted for phase detection. In this method, the input analog rf signal is directly digitized by an analog-to-digital converter (ADC). After digital down conversion (DDC), the in-phase and quadrature $(I Q)$ components of the rf signals are obtained [8].

In the direct sampling technique, the sampling rate $f_{s}$ and the measured rf signal $f_{r f}$ must satisfy [8-10]

$$
f_{s}=\frac{f_{r f}}{K+\frac{N}{M}}, \quad K=0,1,2,3 \ldots,
$$

where $M$ is an integer greater than $3, N$ and $K$ are integers, and $M$ and $N$ are prime to each other. The phase advance $\Delta \varphi$ between the two adjacent samples can be represented as

$$
\Delta \varphi=\left(K+\frac{N}{M}\right) \cdot 2 \pi=\frac{f_{r f}}{f_{s}} \cdot 2 \pi
$$

The $I$ and $Q$ components are calculated by [10]

$$
I=\frac{2}{M} \sum_{n=0}^{M-1} x[n] \cos (\Delta \varphi \cdot n),
$$

$$
Q=-\frac{2}{M} \sum_{n=0}^{M-1} x[n] \sin (\Delta \varphi \cdot n),
$$

where $x[n]$ is the $n$th ADC sampled data. Hence, the $\mathrm{rf}$ phase is obtained by $\phi=\arctan (Q / I)$.

In our case, two different frequency signals should be detected by the same sampling rate. The sampling frequency should satisfy

$$
\begin{aligned}
& \frac{f_{1}}{f_{s}}=K_{1}+\frac{N_{1}}{M_{1}}, \\
& \frac{f_{2}}{f_{s}}=K_{2}+\frac{N_{2}}{M_{2}},
\end{aligned}
$$

where subscripts 1 and 2 denote LMO and RMO, respectively. The frequency ratio of LMO $(571.2 \mathrm{MHz})$ and $\mathrm{RMO}(508.9 \mathrm{MHz})$ is $55: 49$; hence, $f_{2}$ can be expressed by $f_{1}$ as

$$
\frac{f_{2}}{f_{s}}=\frac{49}{55} \cdot \frac{f_{1}}{f_{s}}=\frac{49}{55} \cdot\left(K_{1}+\frac{N_{1}}{M_{1}}\right) .
$$

Under the condition that $K_{1} M_{1}+N_{1}=55$, we have $f_{1} / f_{s}=55 / M_{1}$ and $f_{2} / f_{s}=49 / M_{1}$.

\section{SAMPLING CLOCK GENERATION}

Figure 2 shows the block diagram of the sampling clock generation for the direct sampling technique $[11,12]$. First, the rf signal $\left(f_{r f}\right)$ generated by MO is divided by $X$ to obtain $f_{r f} / X$. Then, $f_{r f} / X$ is mixed with $f_{r f}$ to obtain 


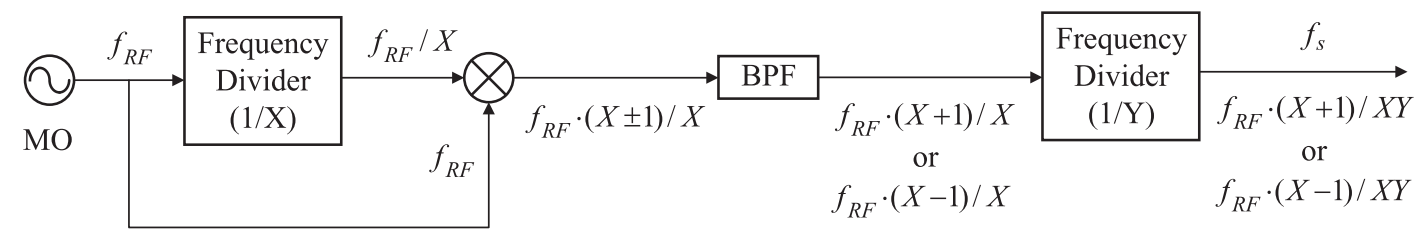

FIG. 2. Block diagram of sampling clock generation for direct sampling technique.

another frequency $f_{r f} \cdot(X \pm 1) / X$. This output frequency is filtered by a band-pass filter (BPF) to obtain the frequency $f_{r f} \cdot(X+1) / X$ or $f_{r f} \cdot(X-1) / X$. Finally, the selected frequency is divided by $Y$ using another frequency divider to generate the required sampling rate $f_{s}$, which can be represented as

$$
f_{s}=\frac{f_{r f}}{\frac{X Y}{X \pm 1}}
$$

where $X$ and $Y$ are both integers.

In our case, the sampling clock is generated based on LMO. From simultaneous Eqs. (5) and (8), it can be concluded that $K_{1} M_{1}+N_{1}=X Y=55$ and $M_{1}=X \pm 1$. Thus, $X$ and $Y$ are either 5 or 11. The possible combinations of the sampling rate are summarized in Table I. According to the non-IQ algorithm [13], the coefficients $K_{1,2}, N_{1,2}$, and $M_{1,2}$ for IQ estimation are listed in Table II. Combination 2 with $M_{1}=2$ and $N_{1}=1$ does not satisfy the IQ sampling condition.

The sampling clock is generated by two frequency dividers (AD9516, Analog Devices, Inc.); the ADC performance is very sensitive to the clock jitter. According to the non-IQ algorithm, the clock-jitter-induced measurement errors can be digitally reduced by averaging over $M$ samples [8]. Thus, the $124.63 \mathrm{MHz}$ sampling rate is chosen for the phase monitor system owing to lower jitter and larger $M\left(M_{1}=M_{2}=12>6,4\right)$. The integral jitter of the

TABLE I. Possible combinations of the sampling rate.

\begin{tabular}{lcrrrc}
\hline \hline Combination & Mixer & \multicolumn{1}{c}{$X$} & \multicolumn{1}{c}{$Y$} & $M_{1}$ & Clock [MHz] \\
\hline 1 & + & 11 & 5 & 12 & 124.63 \\
2 & - & 11 & 5 & 10 & 103.85 \\
3 & + & 5 & 11 & 6 & 63.31 \\
4 & - & 5 & 11 & 4 & 41.54 \\
\hline \hline
\end{tabular}

TABLE II. Possible combinations for the coefficients of non-IQ algorithm for LMO and RMO.

\begin{tabular}{lrrrrrr}
\hline \hline Combination & $M_{1}$ & $K_{1}$ & $N_{1}$ & $M_{2}$ & $K_{2}$ & $N_{2}$ \\
\hline 1 & 12 & 4 & 7 & 12 & 4 & 1 \\
2 & 2 & 5 & 1 & 10 & 4 & 9 \\
3 & 6 & 9 & 1 & 6 & 8 & 1 \\
4 & 4 & 13 & 3 & 4 & 12 & 1 \\
\hline \hline
\end{tabular}

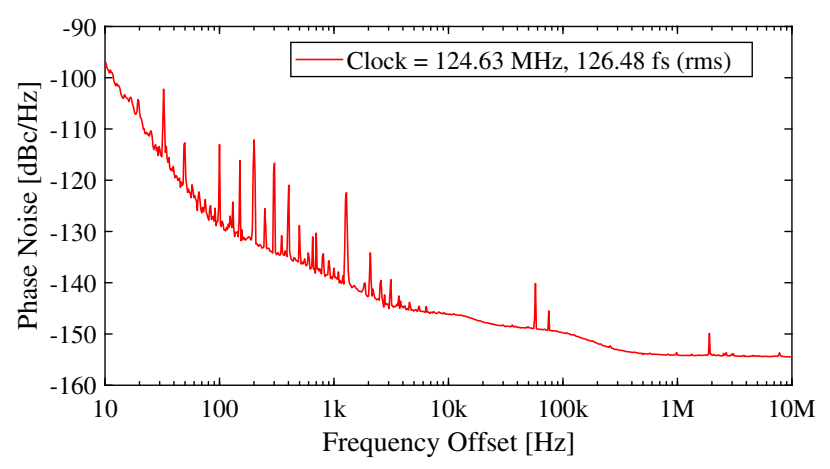

FIG. 3. SSB phase noise power spectrum of sampling clock.

sampling clock is measured with the signal source analyzer (E5052B, Agilent Technologies) in the laboratory and then applied to the injector linac. Figure 3 shows the single side band (SSB) phase noise power spectrum of the $124.63 \mathrm{MHz}$ sampling clock. The integral jitter is $126.48 \mathrm{fs}$ corresponding to 5.7 millidegrees from $10 \mathrm{~Hz}$ to $10 \mathrm{MHz}$. The center frequency of the BPF used for the clock generation is $623 \mathrm{MHz}$ and the bandwidth is $\pm 10 \mathrm{MHz}$. It is a waterproof-type BPF that relatively reduces the humidity effect on the phase monitor system. It is customized by Sogo Electronics, Inc.

\section{PHASE MONITOR SYSTEM}

The configuration of the phase monitor system is shown in Fig. 4. The $571.2 \mathrm{MHz}$ signal generated by the signal generator is split in two: one is for the sampling clock generation and the other is fed to the MO phase shifter (MOPS) used for the beam injection phase adjustment to HER or LER [14]. The output signal of MOPS is monitored. The MOPS and phase monitor system are situated inside the temperature stabilized chamber with $28 \pm 0.1^{\circ} \mathrm{C}$ to reduce the phase drift of the rf components.

The $r f$ reference signal of the RMO is delivered from the DR control room to the linac gallery through the $372 \mathrm{~m}$ phase stabilized optical fiber (PSOF). The PSOF is provided by Furukawa Electric Co., Ltd. [15] with excellent thermal propagation time delay, as low as $0.4 \mathrm{ppm} /{ }^{\circ} \mathrm{C}$ from $25^{\circ} \mathrm{C}$ to $30^{\circ} \mathrm{C}$ [16]. This range covers the temperature changes in the linac gallery. A majority of the PSOF is distributed inside the gallery where the temperature is regulated at $28 \pm 1^{\circ} \mathrm{C}$ by the air conditioner. The PSOF phase drift caused by the temperature fluctuation is estimated 


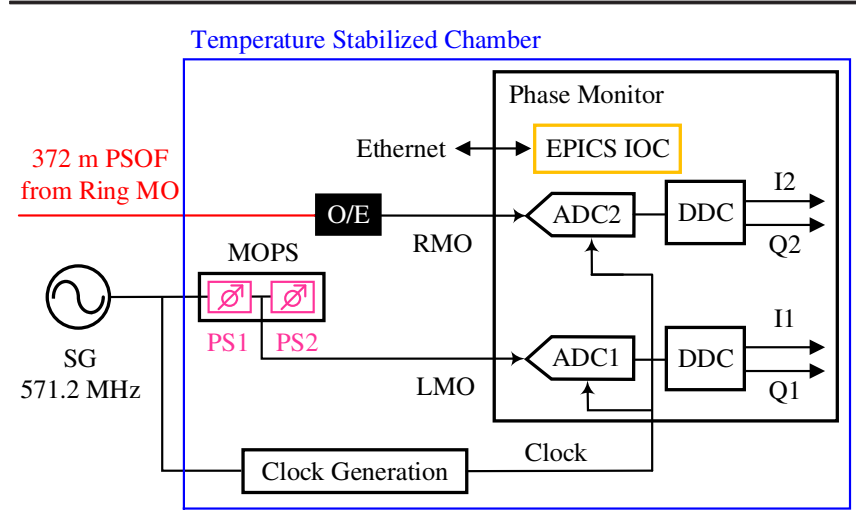

FIG. 4. Phase monitor system for LMO and RMO.

to be less than $1.5 \mathrm{ps}$ (peak to peak) corresponding to $0.27^{\circ}$ for RMO. The optical signal from the RMO is converted to the electrical signal by an optical/electrical converter $(\mathrm{O} / \mathrm{E})$ and then monitored.

The phase monitor was implemented in a digital board based on Micro Telecommunications Computing Architecture (MTCA.0) standard. The same board is used in $[4,12,17,18]$. This board is equipped with Xilinx Virtex5 FXT FPGA, two 14-bit ADCs (400 MSPS high speed, $1.44 \mathrm{GHz}$ input bandwidth, ADS5474) and embedded with an input/output controller application based on Experimental Physics and Industrial Control System (EPICS). The LMO and RMO signals are digitized by ADCs with a $124.63 \mathrm{MHz}$ sampling rate, and then, IQ components are obtained by DDC.

Short-term phase stability without a digital filter is presented in Fig. 5. The phase stabilities of LMO $(571.2 \mathrm{MHz})$ and $\mathrm{RMO}(508.9 \mathrm{MHz})$ are $0.078^{\circ}(\mathrm{rms})$ and $0.064^{\circ}$ (rms), respectively. To improve phase stability, the digital low-pass filter with $100 \mathrm{~Hz}$ bandwidth is applied to suppress the clock jitter and ADC noise [19].

The long-term MO phase difference is shown in Fig. 6. The rf phase of LMO ( $\left.\phi_{\mathrm{LMO571}}\right)$ and RMO $\left(\phi_{\mathrm{RMO} 09}\right)$ is calculated at $571.2 \mathrm{MHz}$ and $508.9 \mathrm{MHz}$, respectively. After the RMO phase is normalized to the LMO frequency, the phase difference $\left(\phi_{\text {DIFF571 }}\right)$ is calculated as

$$
\begin{gathered}
\phi_{\mathrm{RMO} 571}=571.2 / 508.9 \cdot \phi_{\mathrm{RMO} 509}, \\
\phi_{\mathrm{DIFF} 571}=\phi_{\mathrm{RMO571}}-\phi_{\mathrm{LMO} 571},
\end{gathered}
$$
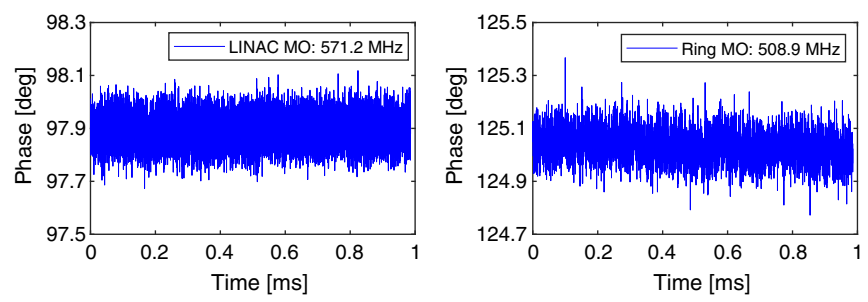

FIG. 5. Short-term phase stability of LMO and RMO.

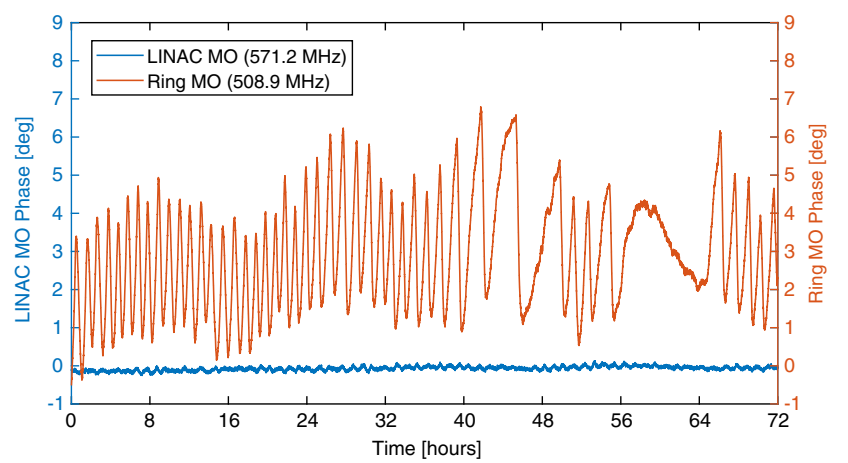

(a) Phase drift of LMO (blue) and RMO (orange).

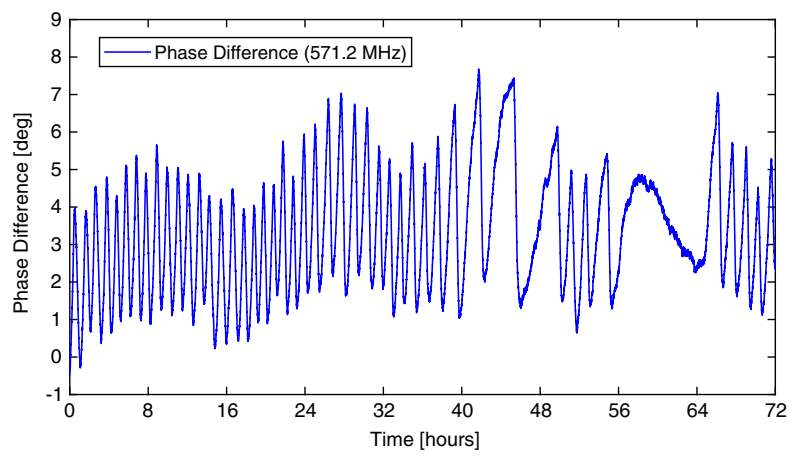

(b) Phase difference between LMO and RMO.

FIG. 6. Phase difference between LMO and RMO for 3 days.

where $\phi_{\mathrm{RMO571}}$ is the RMO phase represented at $571.2 \mathrm{MHz}$.

As shown in Fig. 6(a), the LMO phase drift is $0.39^{\circ}$ (peak to peak) at $571.2 \mathrm{MHz}$ and the RMO phase drift is $7.31^{\circ}$ (peak to peak) at $508.9 \mathrm{MHz}$. Because the sampling clock was generated by LMO, the phase drift of LMO is much smaller than that of RMO. The phase difference between LMO and RMO is observed to be $8.16^{\circ}$ (peak to peak) over 3 days, as shown in Fig. 6(b). The period of the phase difference varies from 1 to $5 \mathrm{~h}$. As mentioned above, the RMO is located in the KEKB control room where the temperature is controlled within $\pm 1^{\circ} \mathrm{C}$ by the air conditioner. However, a strong periodic temperature fluctuation is caused by the working condition of the air conditioner. In addition, the period of this temperature fluctuation matches that of the MO phase difference. Thus, the MO phase difference is correlated with the room temperature of the KEKB control room.

\section{MO PHASE DIFFERENCE EFFECTS ON BEAM PERFORMANCE AT BCS}

The layout of the beam transport lines between the injector linac and the DR is shown in Fig. 7 [20]. Figure 8 shows the horizontal beam positions of two beam position monitors (BPMs). BPM1 is located at the first arc section after DR extraction. BPM2 is located at the second arc section after the $2856 \mathrm{MHz}$ accelerating structure of BCS. There is no slow drift at BPM1; however, it shows a clear 


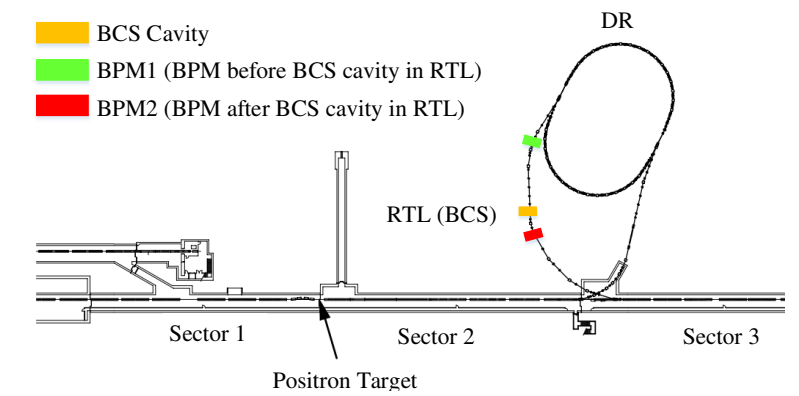

FIG. 7. Layout of beam transport lines between injector linac and DR. Locations of two BPMs are marked in the beam transportation line.

slow drift at BPM2. As shown in Fig. 7, the extracted positron beam from the DR is accelerated by the BCS cavity operated at $2856 \mathrm{MHz}$. This $2856 \mathrm{MHz}$ signal is generated by LMO, but the rf of the DR is provided by the RMO. It is found out that the slow drift of horizontal beam position at BPM2 depends on the phase difference between LMO and RMO.

To confirm the relationship between the BCS rf phase and the horizontal beam position, the rf phase was scanned. Figure 9 shows the measurement result. The slope of the linear fitting equation is $-0.517 \mathrm{~mm} /{ }^{\circ}$ at $2856 \mathrm{MHz}$. In Fig. 8, the slow drift of the horizontal beam position is
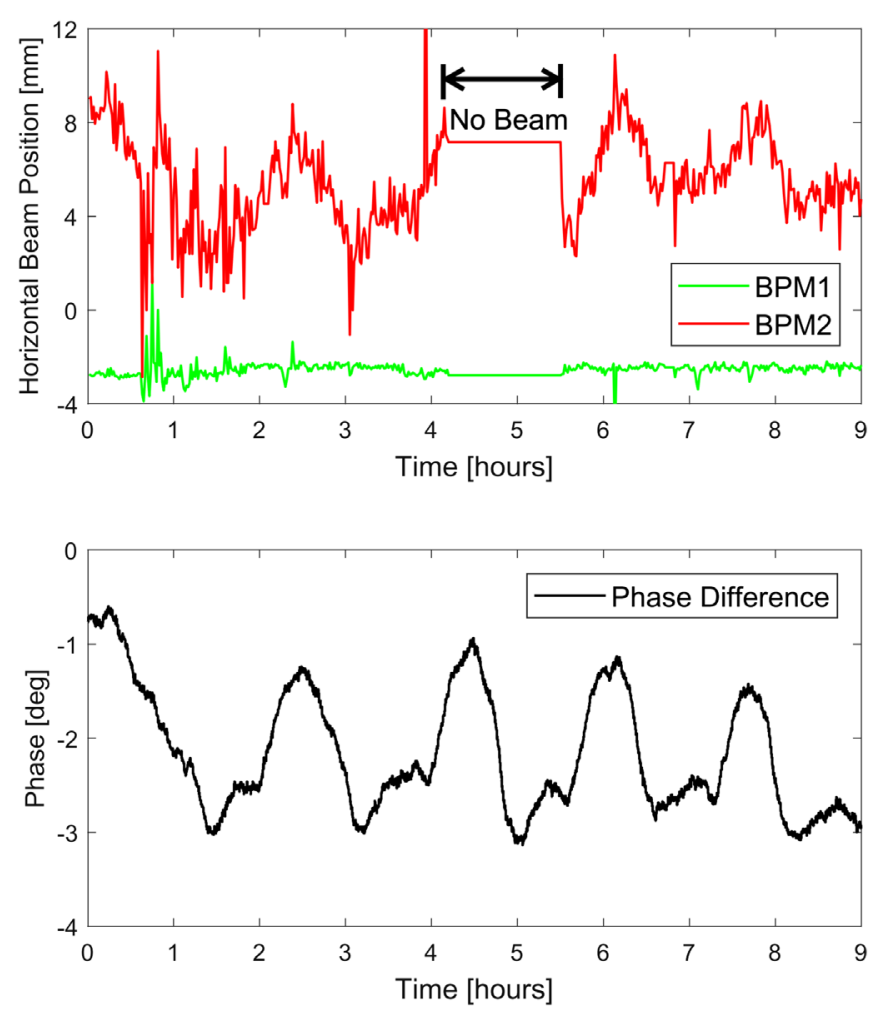

FIG. 8. Horizontal beam position at BPM1 and BPM2 (top) and the phase difference between LMO and RMO (bottom) without phase drift compensation system for $9 \mathrm{~h}$.

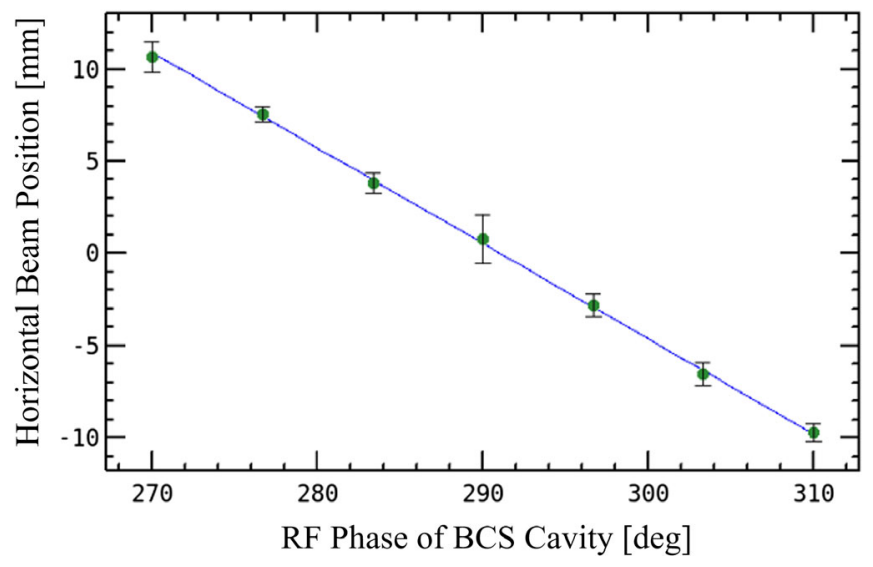

FIG. 9. Horizontal beam position as a function of $2856 \mathrm{MHz}$ rf phase of BCS cavity. The slope is $-0.517 \mathrm{~mm} /{ }^{\circ}$.

observed approximately at $6 \mathrm{~mm}$. The $6 \mathrm{~mm}$ deviation corresponds to $11.6^{\circ} \mathrm{BCS}$ phase change at $2856 \mathrm{MHz}$ (peak to peak). Meanwhile, the observed MO phase difference is $2.3^{\circ}$ at $571.2 \mathrm{MHz}$ (peak to peak) which corresponds to $11.5^{\circ}$ at $2856 \mathrm{MHz}$. These two results are consistent. The $\mathrm{MO}$ phase difference is too large to satisfy the requirement $\pm 0.1^{\circ}$ (peak to peak) at $571.2 \mathrm{MHz}$ so that the phase drift compensation system is developed.

\section{PHASE DRIFT COMPENSATION}

The linac rf phase should be shifted based on the injection mode of HER and LER at the $50 \mathrm{~Hz}$ repetition rate. However, a fast phase change is not allowed for the laser system of the photo cathode rf gun, as shown in Fig. 1. MOPS is installed to satisfy the requirement for the laser system and the injection phase adjustment [14]. MOPS consists of two phase shifters, PS1 and PS2. PS1 is located upstream and fixed at the HER injection phase. The PS1 output signal is sent to the laser system. PS2 is located downstream and is changed every $20 \mathrm{~ms}$ depending on injection modes. For MO phase drift compensation, the LMO phase is changed to follow the RMO phase by using MOPS.

As mentioned above, MOPS is not only used for beam injection phase adjustment but also phase drift compensation. Thus, the monitored phase drift of LMO includes the injection phase change. For phase drift evaluation, the amount of injection phase change is removed by calculation. The MO phase difference is fed back to MOPS through the EPICS record. A proportional integral (PI) controller is applied for precise feedback control. Figure 10 shows the long-term phase difference between LMO and RMO with compensation. The LMO successfully follows $\mathrm{RMO}$ and the phase difference between them is compensated within $0.018^{\circ}(\mathrm{rms})$ at $571.2 \mathrm{MHz}$, which fulfilled the phase stability requirement $\pm 0.1^{\circ}$ (peak to peak).

The horizontal beam position results with the MO phase drift compensation are shown in Fig. 11. The large slow 


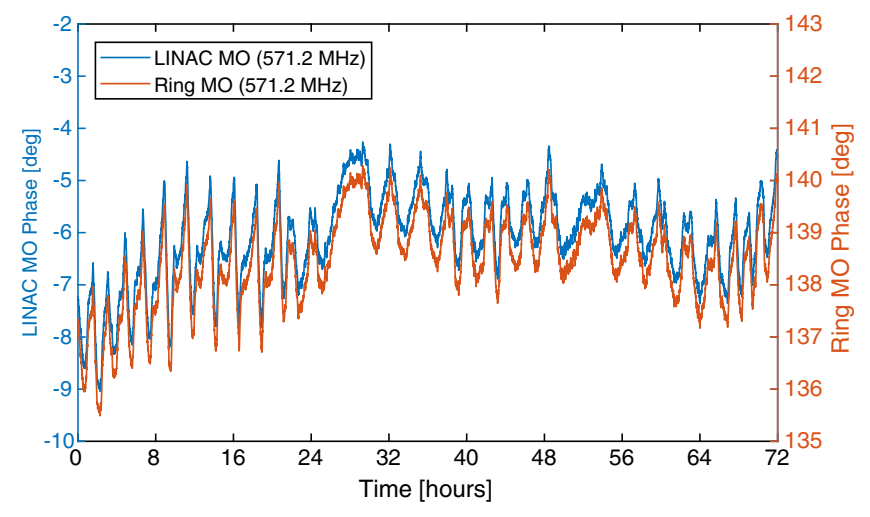

(a) Phase drift of LMO (blue) and RMO (orange).

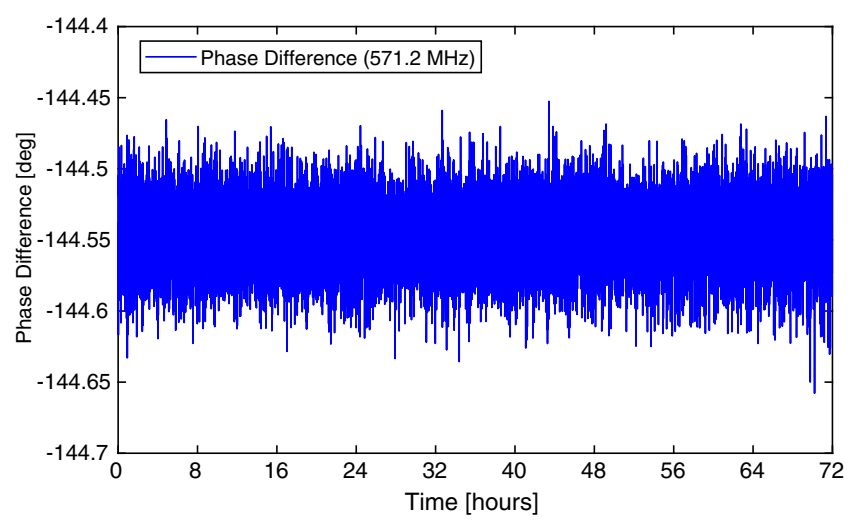

(b) Phase difference between LMO and RMO.

FIG. 10. Phase difference between LMO and RMO for 3 days with compensation.

drift of the horizontal beam position at BPM2 is compensated. The energy jitter with the stability of $0.7 \mathrm{~mm}$ (rms) still remains; however, it is not caused by the MO phase difference. The possible sources are the klystron high voltage fluctuation, the LLRF control unit, and the measurement error of BPM2. Thus, the slow drift of the beam energy is stabilized by the MO phase drift compensation system.

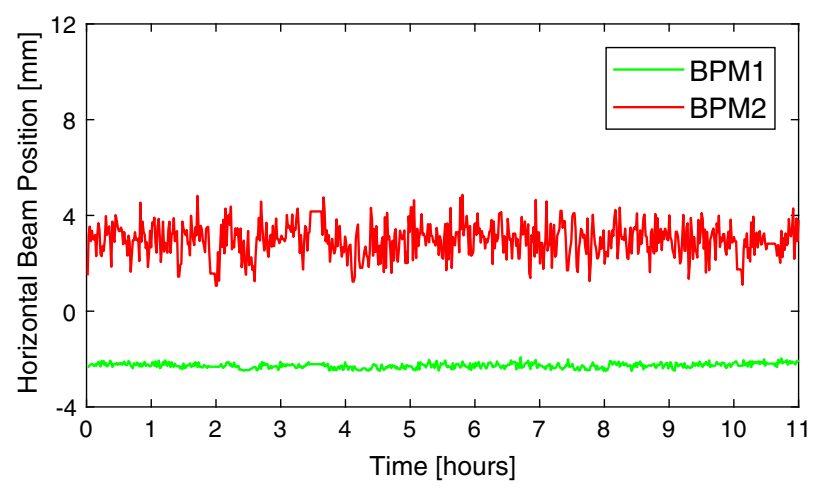

FIG. 11. Horizontal beam position at BPM1 and BPM2 with the phase drift compensation system for 11 hours.

\section{SUMMARY}

The LMO (571.2 MHz) and RMO (508.9 MHz) signals are monitored by the direct sampling technique with a common sampling rate. A large fluctuation of the phase difference between the two MOs is observed by the phase monitor system. The phase drift compensation system is developed and used in the SuperKEKB operation. The MO phase difference is stabilized within $0.018^{\circ}(\mathrm{rms})$ at 571.2 MHz, which fulfilled the requirement. The slow drift of the beam energy after the BCS cavity caused by the MO phase drift is suppressed. It is also very helpful for the stable beam injection to the MR and the machine operation.

[1] Y. Ohnishi et al., Accelerator design at SuperKEKB, Prog. Theor. Exp. Phys. (2013), 03A011.

[2] M. Akemoto et al., The KEKB injector linac, Prog. Theor. Exp. Phys. (2013), 03A002.

[3] T. Kobayashi et al., LLRF control and master oscillator system for damping ring at SuperKEKB, in Proceedings of the International Particle Accelerator Conference (IPAC'2018), Vancouver, Canada (JACoW, Vancouver, Canada, 2018), WEPAL001, p. 2137.

[4] T. Kobayashi et al., RF reference distribution system for SuperKEKB, in Proceedings of the 10th Annual Meeting of Particle Accelerator Society of Japan (PASJ'2013), Nagoya, Japan (Japan Accelerator Society, Nagoya, Japan, 2013), SUP094, p. 1159.

[5] T. Matsumoto et al., Low-lever rf system for the SuperKEKB injector LINAC, in Proceedings of the International Particle Accelerator Conference (IPAC'2018), Vancouver, Canada (JACoW, Vancouver, Canada, 2018), WEPAK017, p. 2132.

[6] H. Hayano and E. Ezura, The rf reference line for TRISTAN, in Proceedings of the IEEE Particle Accelerator Conference (PAC'1987), Washington (JACoW, Washington, 1987), p. 1663.

[7] P. M. Lewis et al., First measurements of beam backgrounds at SuperKEKB, Nucl. Instrum. Methods Phys. Res., Sect. A 914, 69 (2019).

[8] T. Schilcher, RF application in digital signal processing, in Proceedings of the CERN Accelerator School on Digital Signal Processing, (CAS2007), Sigtuna, Sweden, 2007 (CERN, Geneva, 2008), p. 249.

[9] Z. Geng and S. N. Simrock, Evaluation of fast ADCs for direct sampling rf filed detection for the European XFEL and ILC, in Proceedings of the Linear Accelerator Conference (LINAC'2008), Victoria, Canada (JACoW, Victoria, Canada, 2008), THP102, p. 1030.

[10] M. Grecki, T. Jezynski, and A. Brandt, Estimation of IQ vector components of rf field theory and implementation, in Proceedings of the 12th Mixed Design of Integrated Circuits and Systems (MIXDES'05, Krakow, Poland, 2005), p. 783.

[11] T. Matsumoto et al., Simultaneous measurement of $\mathrm{rf}$ signal and LO signal using direct sampling detection technique, in Proceedings of the 14th Annual Meeting of Particle Accelerator Society of Japan (PASJ'2017), Hokkaido, Japan (Japan Accelerator Society, Hokkaido, Japan, 2017), TUP063, p. 493. 
[12] S. B. Wibowo, T. Matsumoto, S. Michizono, T. Miura, F. Qiu, and N. Liu, Digital low level rf control system for the International Linear Collider, Phys. Rev. Accel. Beams 21, 082004 (2018).

[13] L. Doolittle, Digital low-level rf control using non-IQ sampling, in Proceedings of the Linear Accelerator Conference (LINAC'2006), Knoxville, TN (JACoW, Knoxville, USA, 2006), THP004, p. 568.

[14] T. Miura et al., Phase shifter for rf reference signal in SuperKEKB injector LINAC, in Proceedings of the 15th Annual Meeting of Particle Accelerator Society of Japan (PASJ'2018), Nagaoka, Japan (Japan Accelerator Society, Nagaoka, Japan, 2018), THP106, p. 1136.

[15] Furukawa Electric, https://www.furukawa.co.jp/en.

[16] T. Kobayashi et al., RF reference distribution system for the 400-MeV proton linac of the KEK/JAERI joint project, in Proceedings of the Linear Accelerator Conference (LINAC'2002), Gyeongju, Korea (JACoW, Gyeongju, Korea, 2002), MO463, p. 187.
[17] J. Mizuno et al., A new FPGA board with fast ADCs for direct rf sampling, in Proceedings of the 8th Annual Meeting of Particle Accelerator Society of Japan (PASJ'2011), Tsukuba, Japan (Japan Accelerator Society, Tsukuba, Japan, 2011), MOPS106, p. 609.

[18] T. Miura et al., Low-level rf system for cERL, in Proceedings of the International Particle Accelerator Conference (IPAC'2010), Kyoto, Japan (JACoW, Kyoto, Japan, 2010), TUPEA048, p. 1441.

[19] F. Qiu et al., Progress in the work on the tuner control system of the cERL at KEK, in Proceedings of the International Particle Accelerator Conference (IPAC'2016), Busan, Korea (JACoW, Busan, Korea, 2016), WEPOR033, p. 2743.

[20] N. Iida et al., Design of the positron transport system for SUPER-KEKB, in Proceedings of the International Particle Accelerator Conference (IPAC'2010), Kyoto, Japan (JACoW, Kyoto, Japan, 2010), THPD004, p. 4284. 